\title{
EFEITO DA TEMPERATURA E CONCENTRAÇÃO NAS PROPRIEDADES TERMOFÍSICAS DE SOLUÇÕ̃ES AQUOSAS CONTENDO PECTINA E CARBOXIMETILCELULOSE
}

\author{
B. L. L. D. SILVA ${ }^{1}$, A. J. SILVA ${ }^{1}$, B. S. $\operatorname{COSTA}^{2}$ e E. E. G. ROJAS ${ }^{2}$ \\ ${ }^{1}$ Universidade Federal Fluminense, Pós Graduação em Engenharia Mecânica \\ ${ }^{2}$ Universidade Federal Fluminense, Departamento de Engenharia de Agronegócios \\ E-mail para contato: edwin@vm.uff.br
}

\begin{abstract}
RESUMO - Os polímeros têm sido foco de intensa pesquisa por sua grande aplicabilidade em vários segmentos industriais. Entre os principais polímeros estão a carboximetilcelulose e a pectina. Este trabalho representa uma contribuição ao estudo das propriedades termofísicas das soluções aquosas binárias, ternárias e pontos de mistura dos sistemas Pectina-CMC. A massa específica $(\rho)$, índices de refração (n) e viscosidades ( $\eta$ ) das soluções foram obtidas para diversas temperaturas e concentrações. Foi verificado que as propriedades termofísicas sofreram elevação com o aumento das concentrações e redução com o aumento das temperaturas. Os dados experimentais foram correlacionados empregando o software estatístico SAS ${ }^{\circledR}$ para obtenção de equações que descrevam o efeito combinado da temperatura e da concentração sobre as propriedades termofisicas. $\mathrm{O}$ modelo polinomial empregado apresentou um bom ajuste aos dados experimentais.
\end{abstract}

\section{INTRODUÇÃO}

Os biopolímeros têm sido objeto de intensa pesquisa, tendo em vista o seu alto potencial de aplicação em vários segmentos industriais. Inúmeras aplicações de soluções de biopolímeros são encontradas na indústria de alimentos, farmacêutica, de cosméticos, de tintas e vernizes, de processamento de petróleo e de mineração (KREVELEN, 1990). Entre os principais biopolímeros estão a pectina e a carboximetilcelulose (CMC).

A Carboximetilcelulose (CMC), obtida através da reação da celulose com monocloroacetato de sódio, é um hidrocolóide que contribui para formação de gel e na retenção de água (KÄISTNER et al., 1997). A estrutura da Carboximetilcelulose é baseada no polímero de $\beta$-(1,4)-D-glucopiranose da celulose e possui uma ampla aplicação, tanto na formulação de produtos alimentícios, quanto no melhoramento de seus processamentos. (ALHAMDAN e SASTRY, 1990; PILIZOTA et al.,1996). A pectina pertence ao grupo de heteropolissacarídeos e são encontradas nas paredes celulares primárias das plantas (PHILIPS e WILLIAMS, 2009). A pectina é um polissacarídeo com cerca de 150 a 500 unidades de ácidos galacturônicos parcialmente estereficados com grupos metoxilícos, unidos por ligações glicosídicas $\alpha-1,4$ em uma longa cadeia molecular (BOBBIO, 1989). As matérias-primas mais importantes para a extração comercial de pectina constituem-se na polpa de maçã e cascas de frutas cítricas, 


\section{9 a 22 de outubro de 2014 \\ Florianópolis/SC}

subprodutos da indústria de sucos (THAKUR et al., 1997). Elas são utilizadas em alimentos, como espessantes, texturizantes, emulsificantes ou estabilizantes (BOWERS, 1992).

As soluções formadas por biopolímeros têm importância tanto pelo aspecto científico quanto tecnológico, portanto, é essencial o conhecimento das propriedades termofísicas destas soluções (KREVELEN, 1990). O conhecimento das propriedades termofísicas é muito importante para cálculos de engenharia que envolvam a seleção e dimensionamento de equipamentos, assim como, a implementação de estratégias de controle de processos (McCABE et al., 1993).

O presente trabalho tem por objetivo de determinar as propriedades termofísicas de soluções binárias e ternárias de Carboximetilcelulose e Pectina em função da temperatura e concentração.

\section{MATERIAIS E MÉTODOS}

\subsection{Materiais}

As soluções foram preparadas utilizando pectina obtida de cascas cítricas (Número do Produto P9135; Galacturonic acid $\geq 74.0 \%$ ), carboximetilcelulose sódica de média viscosidade (Número do Produto C4888; pureza $\approx 99,5 \%$ ) e água ultrapura (condutividade $\approx 0,05 \mu \mathrm{S} / \mathrm{cm}$; resistividade elétrica $\approx 18.2 \mathrm{M} \Omega \cdot \mathrm{cm}$; Master System P\&D Gehaka, Brazil). Os biopolímeros utilizados no estudo foram adquiridos da Sigma-Aldrich (St.Louis, USA). Todos os experimentos foram realizados em três repetições.

\subsection{Métodos}

Preparo das soluções aquosas. As soluções aquosas estudadas neste trabalho foram preparadas diluindo as devidas concentrações de biopolímeros em água ultrapura. No preparo das soluções foi utilizado uma balança analítica (Modelo AY220, Shimadzu, Japão) com uma incerteza de $\pm 0,0001 \mathrm{~g}$. As soluções foram submetidas à agitação magnética (78 $H W-1$, Biomixer, Brasil) por 3 horas. Após homogeneização, aplicou-se o ultrassom por 30 minutos (Ultrassonic Processor UP100H, Hielscher, Alemanha) à amplitude de 100\% e ciclo de 0,5/min.

Construção do diagrama de fases. Para construção do diagrama de equilíbrio do sistema CMC-Pectina foram elaboradas misturas de soluções aquosas binárias de carboximetilcelulose e pectina a diferentes concentrações. Essas misturas foram submetidas à centrífuga refrigerada (Orto-Alresa, Modelo Digicen 21R, Espanha) por 30 minutos a uma temperatura de 283,15 K e rotação de 8500 RPM, observou-se então a existência ou não de duas fases nas amostras.

Análise das massas específicas. As massas específicas $(\rho)$ das amostras foram observadas com o auxílio do densímetro digital DMA 4500M (Anton Paar, Graz, Austria) com uma incerteza de $\pm 1.0 \cdot 10^{-5} \mathrm{~g} \cdot \mathrm{cm}^{-3}$ em temperaturas na faixa de 298,15 a $308,15 \mathrm{~K}$. O densímetro 
digital foi acoplado ao trocador de amostras automático Xsample 122 da Anton Paar (Graz, Austria).

Análise dos índices de refração. Os índices de refração $(n)$ das amostras foram obtidos utilizando o refratômetro digital Abbemat RXA170 integrado ao densímetro digital DMA 4500M (Anton Paar, Graz, Austria) em temperaturas entre 298,15 e 308,15 K. A precisão do densímetro digital é de $\pm 1.0 \cdot 10^{-5} \mathrm{~g} \cdot \mathrm{cm}^{-3}$ e a precisão do refratômetro digital é de $\pm 4.0 \cdot 10^{-5}$. Ambos os instrumentos foram conectados a um trocador de amostras automático (Xsample 122, Anton Paar, Graz, Austria).

Análise das viscosidades dinâmicas. As viscosidades dinâmicas das amostras foram determinadas através de ensaios realizados com o reômetro rotacional Thermo Haake MARS (Modular Advanced Rheometer System), utilizando geometria cone (modelo C60/1 ${ }^{\circ} \mathrm{Ti}$ L) e placa ( modelo MPC 60) nas temperaturas de 298,15 a 308,15K. Os dados obtidos no ensaio foram visualizados através do software HAAKE RheoWin Job Manager (versão 4.41.0000) e manipulados com o auxílio do software HAAKE RheoWin Data Manager (versão 4.41.0000).

Modelagem dos dados. Os dados experimentais foram analisados utilizando o pacote estatístico SAS ${ }^{\circledR}$, Statistical Analysis System, versão 9.0 (SAS Institute Inc., Cary, NC). Adotouse, como critério de análise das variáveis dos modelos, o nível de significância de $5 \%(\mathrm{p}<0,05)$. A equação 1 demonstra o modelo quadrático geral utilizado neste estudo,

$$
\Psi=\beta_{1}+\beta_{2} T+\beta_{3} C_{1}+\beta_{4} C_{2}+\beta_{5} T^{2}+\beta_{6} C_{1}^{2}+\beta_{7} C_{2}^{2}+\beta_{8} C_{1} T+\beta_{9} C_{2} T+\beta_{10} C_{1} C_{2}
$$

onde, $\Psi$ é a propriedade termofísica $, \beta_{1}, \beta_{2}, \beta_{3}, \beta_{4}, \beta_{5}, \beta_{6}, \beta_{7}, \beta_{8}, \beta_{9}$ e $\beta_{10}$ são constantes determinadas a partir do ajuste dos dados experimentais, T é a temperatura, e $C_{1}$ e $C_{2}$ são as frações mássicas dos biopolímeros pectina e carboximetilcelulose respectivamente.

\section{RESULTADOS E DISCUSSÃO}

\subsection{Diagrama de Fases}

Através das análises da presença ou ausência de duas fases nas soluções submetidas à centrífuga refrigerada obteve-se os diagramas de equilíbrio dos sistemas estudados. $\mathrm{O}$ diagrama construído para o sistema CMC-Pectina pode ser observado a seguir (Figura 1): 


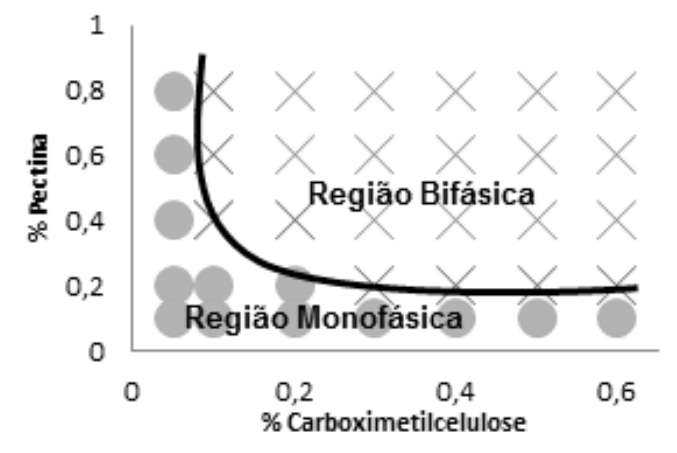

Figura 1 - Diagrama de Equilíbrio de Fases do Sistema Pectina - Carboximetilcelulose.

As misturas das soluções binárias com as concentrações indicadas que estejam acima da linha binoidal serão denominadas neste trabalho por soluções aquosas ternárias bifásicas ou pontos de mistura e abaixo da linha binoidal, soluções aquosas ternárias monofásicas. Perrechil e Cunha (2012) utilizaram este mesmo método para determinar o diagrama de equilíbrio de fases do sistema Caseína-LBG, para eles, as condições necessárias para a separação de fases variam de acordo com as propriedades específicas das macromoléculas dos biopolímeros, com as características da composição das soluções e com a temperatura.

\subsection{Massa Específica, Índice de Refração e Viscosidade Dinâmica}

Segundo o diagrama de fases definiu-se as concentrações a serem estudadas. As massas específicas, índices de refração e viscosidades dinâmicas de soluções aquosas binárias do sistema Pectina-CMC foram analisadas em concentrações na faixa de 0,10 a 1,00\% de pectina e 0,05 a $0,60 \%$ de carboximetilcelulose. No caso das soluções aquosas ternárias ou pontos de mistura foram estudadas as combinações de pectina e CMC contendo $0,10 \%$ a $0,80 \%$ de pectina e a concentração de CMC variando de $0,05 \%$ a $0,70 \%$ das soluções. Todas as medidas foram realizadas em temperaturas entre 298,15 e $308,15 \mathrm{~K}$.

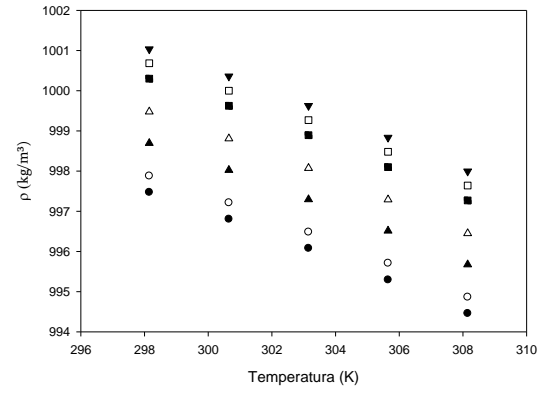

(a)

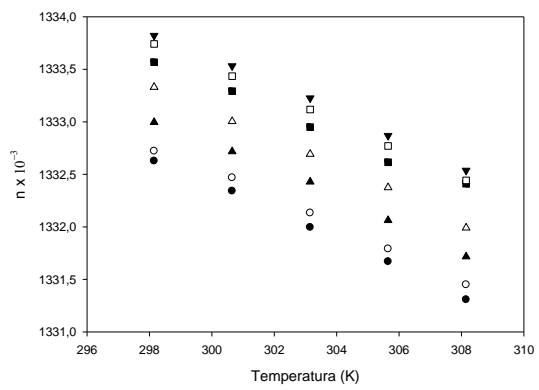

(b)

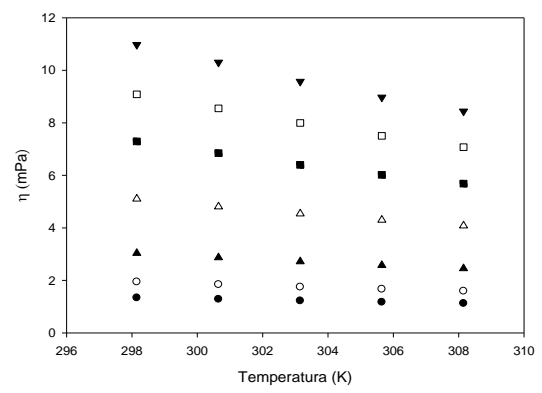

(c)

Figura 2 - Massa específica (a), índice de refração (b) e viscosidade dinâmica (c) das soluções binárias de pectina em função da temperatura para diferentes concentrações de pectina: 0,10 $\%(\bullet) ; 0,20 \%(\circ) ; 0,40 \%(\mathbf{\Delta}) ; 0,60 \%(\Delta) ; 0,80 \%(\bullet) ; 0,90 \%$ (口) e $1,00 \%(\mathbf{\nabla})$. 


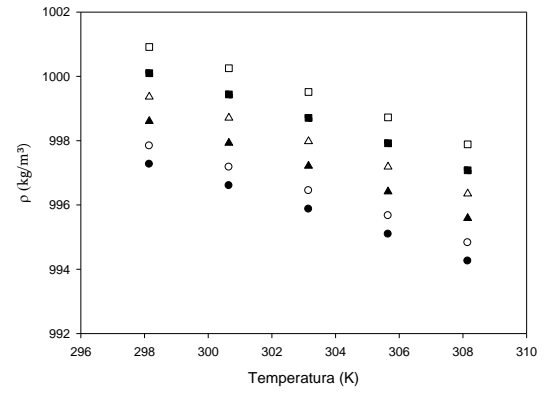

(a)

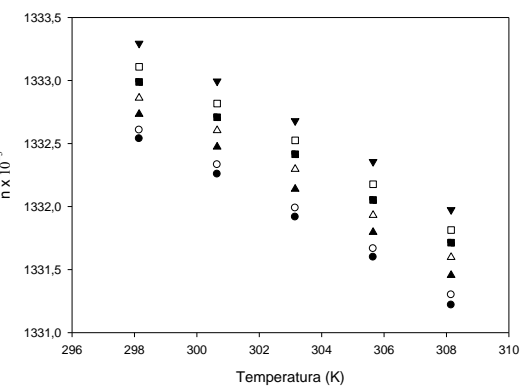

(b)

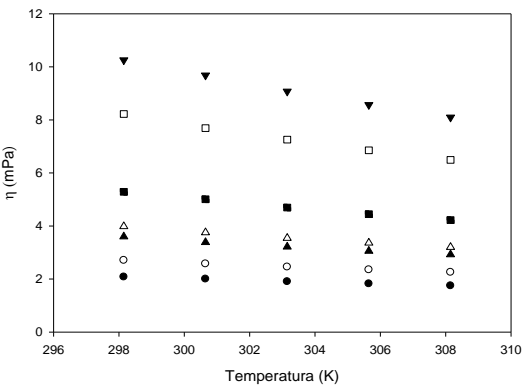

(c)

Figura 3 - Massa específica (a), índice de refração (b) e viscosidade dinâmica (c) das soluções binárias de $\mathrm{CMC}$ em função da temperatura para diferentes concentrações de CMC: 0,05\%(•); $0,10 \%(\circ) ; 0,20 \%(\mathbf{\Delta}) ; 0,30 \%(\Delta) ; 0,40 \%(\boldsymbol{\square}) ; 0,50 \%(\square)$ e $1,00 \%(\boldsymbol{\nabla})$.

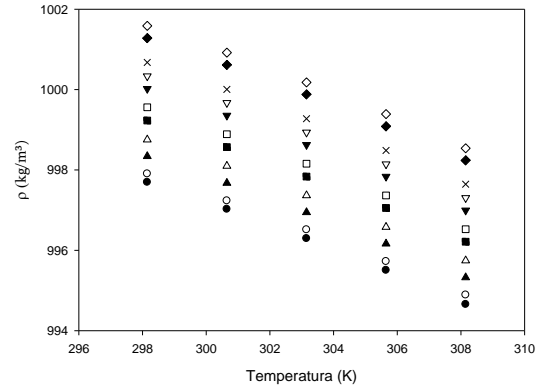

(a)

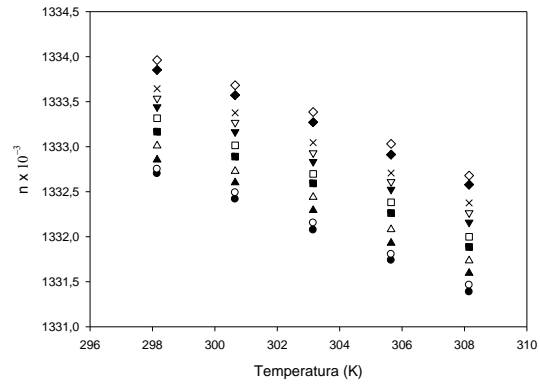

(b)

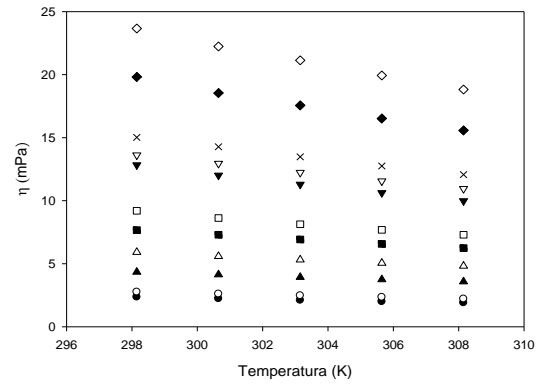

(c)

Figura 4 - Massa específica (a), índice de refração (b) e viscosidade dinâmica (c) das soluções de Pectina-CMC (Região Monofásica) para Pectina 0,10\% e diferentes concentrações de CMC: 0,05 $\%(\bullet) ; 0,10 \%(\circ) ; 0,20 \%(\boldsymbol{\Delta}) ; 0,30 \%(\Delta) ; 0,40 \%(\mathbf{\square}) ; 0,50 \%(\square) ; 0,60 \%(\boldsymbol{\nabla}) ; 0,70 \%(\nabla) ; 0,80 \%$ $(\mathrm{x}) ; 0,90 \%(\diamond)$ e $1,00 \%(\diamond)$.

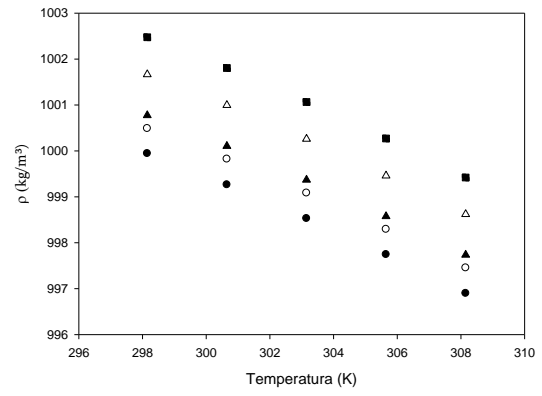

(a)

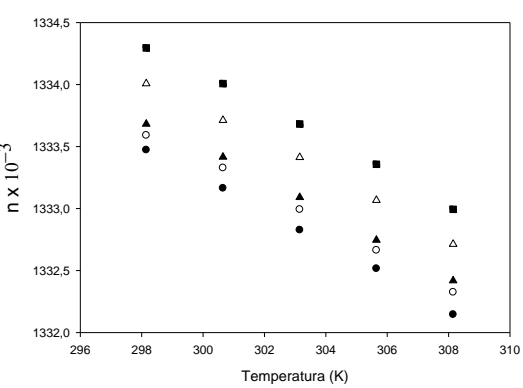

(b)

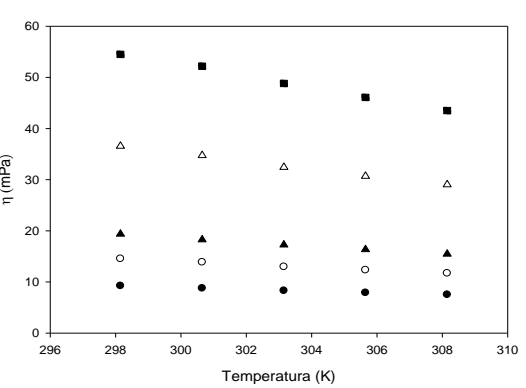

(c)

Figura 5 - Massa específica (a), índice de refração (b) e viscosidade dinâmica (c) das soluções de Pectina-CMC (Região Bifásica): Pectina 0,40\%- CMC 0,30\%(•); Pectina 0,50\%- CMC 0,40\%

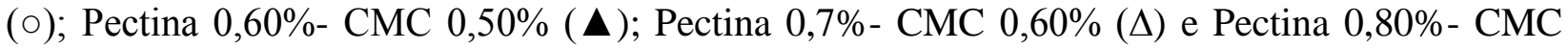
$0,60 \%(\mathbf{\square})$. 


\section{9 a 22 de outubro de 2014 \\ Florianópolis/SC}

A análise das médias dos dados obtidos experimentalmente para a massa específica, nos mostra que a massa específica é elevada com o aumento da concentração e reduzida com o aumento da temperatura. Ao avaliar o índice de refração do sistema estudado, verifica-se que as médias dos dados obtidos experimentalmente apontaram o decréscimo do índice de refração com o aumento da temperatura e a elevação do mesmo com o aumento da concentração. E por último, através dos dados obtidos pode ser observado que a viscosidade dinâmica das soluções foi elevada com o aumento das concentrações dos biopolímeros e reduzida com o aumento da temperatura nas soluções estudadas.

O comportamento apresentado neste estudo foi reportado por outros autores na literatura, tais como Guimarães et al. (2009) e Assis et al. (2010), os quais estudaram as massas específicas e viscosidades dinâmicas de soluções aquosas de pectina e de soluções binárias e ternárias contendo goma xantana, carboximetilcelulose, cloreto de sódio e água, respectivamente, os autores observaram que houve o aumento da massa específica e da viscosidade dinâmica com o aumento da concentração e a redução de ambas as propriedades termofísicas com o aumento de temperatura. $\mathrm{O}$ estudo das massas específicas e das viscosidades também foi realizado por Ramos et al. (2013) para soluções ternárias contendo $\kappa$-carragena, cloreto de sódio e água em diferentes $\mathrm{pHs}$ e temperaturas, eles verificaram que as massas específicas e as viscosidades do sistema sofreram redução com o aumento da temperatura para todos os valores de $\mathrm{pH}$ estudados.

\subsection{Modelagem dos Dados}

Os dados experimentais obtidos na análise das propriedades termofísicas foram verificados através do software estatístico SAS® (método backward) permitindo assim a determinação de modelos empíricos que descrevam o efeito combinado da temperatura e da concentração sobre a variação da massa específica, índice de refração e viscosidade. Considerando os modelos que melhor se ajustaram aos dados experimentais do sistema PectinaCMC determinou-se as equações apresentadas na Tabela 2.

Tabela 2 - Equações dos modelos que melhor se ajustaram aos dados experimentais

\begin{tabular}{|c|c|}
\hline Solução Binária de Pectina & $\mathbf{R}^{2}$ \\
\hline$\rho=681,66+2,375 \mathrm{~T}+396,183 \mathrm{C}_{1}-0,00442 \mathrm{~T}^{2}$ & 0,9999 \\
\hline$n=1266,58+0,561 \mathrm{~T}+12,9677 \mathrm{C}_{1}-0,00114 \mathrm{~T}^{2}-592,7578 \mathrm{C}_{1}^{2}+0,4334 \mathrm{~T} \cdot \mathrm{C}_{1}$ & 0,9982 \\
\hline$\eta=1,144+0,00 \mathrm{~T}+6747,78 \mathrm{C}_{1}+0,00 \mathrm{~T}^{2}+73328,00 \mathrm{C}_{1}{ }^{2}-21,91 \mathrm{~T} \cdot \mathrm{C}_{1}$ & 0,9975 \\
\hline \multicolumn{2}{|l|}{ Solução Binária de CMC } \\
\hline$\rho=663,276+2,494 \mathrm{~T}+429,927 \mathrm{C}_{2}-0,00461 \mathrm{~T}^{2}-5852,757 \mathrm{C}_{2}{ }^{2}$ & 0,9997 \\
\hline$n=1171,85+1,18 \mathrm{~T}+70,07 \mathrm{C}_{2}-0,00217 \mathrm{~T}^{2}-105,65 \mathrm{C}_{2}{ }^{2}+0,209 \mathrm{~T} \cdot \mathrm{C}_{2}$ & 0,9991 \\
\hline$\eta=3,44-0,0042 T+9951,72 C_{2}+0,00 T^{2}+211690,00 C_{2}{ }^{2}-32,18 T \cdot C_{2}$ & 0,9853 \\
\hline \multicolumn{2}{|l|}{ Solução Ternária Pectina-CMC (Região Monofásica e Região Bifásica) } \\
\hline$\rho=662,10+2,505 \mathrm{~T}+378,10 \mathrm{C}_{1}+404,69 \mathrm{C}_{2}+0,00463 \mathrm{~T}^{2}-11019,00$ & 0,9959 \\
\hline$n=1200,60+0,995 \mathrm{~T}+126,89 \mathrm{C}_{1}+129,24 \mathrm{C}_{2}-0,00185 \mathrm{~T}^{2}-3359,86 \mathrm{C}_{1}^{2}+415,48 \mathrm{C}_{2}^{2}$ & 0,9952 \\
\hline$\eta=288,776-2,0166 \mathrm{~T}+18185,00 \mathrm{C}_{1}+17040,00 \mathrm{C}_{2}+0,00359 \mathrm{~T}^{2}+1202518,00 \mathrm{C}_{1}^{2}+126205,00 \mathrm{C}_{2}^{2}$ & \\
\hline$-79,1768 \mathrm{~T} \cdot \mathrm{C}_{1}-54,3575 \mathrm{~T} \cdot \mathrm{C}_{2}$ & 0,9857 \\
\hline
\end{tabular}


Ramos et al. (2013), obtiveram modelos similares aos apresentados neste estudo para determinar como soluções binárias e ternárias contendo $\kappa$-carragena, cloreto de sódio e água variam em função da temperatura e concentração. Modelos polinomiais quadráticos para expressar o efeito da temperatura e concentração sobre as propridades termofísicas também foram construídos por Costa et al. (2014), quando os mesmos estudaram soluções de PEG1500.

Considerando os elevados valores dos coeficientes de determinação $\left(\mathrm{R}^{2}\right)$, os modelos propostos podem ser satisfatoriamente utilizados para descrever a variação das propriedades físicas com a concentração e a temperatura simultaneamente, e pode ser de grande utilidade em cálculos de engenharia.

\section{CONCLUSÃO}

Os sistemas foram analisados em diversas temperaturas considerando diferentes concentrações poliméricas. Observou-se, através dos dados obtidos com os experimentos, que as massas específicas, índices de refração e viscosidades sofreram elevação com o aumento das concentrações e redução com o aumento das temperaturas, como esperado.

Os modelos empíricos, obtidos através de análise das médias dos dados experimentais com o auxílio do pacote estatístico $\mathrm{SAS}^{\circledR}$, mostraram como que as propriedades termofísicas variaram com a temperatura e concentração. Os modelos empíricos expostos neste estudo apresentaram elevados coeficientes de determinação $\left(R^{2}>0,98\right)$ podendo ser satisfatoriamente utilizados em cálculos de engenharia.

\section{REFERÊNCIAS}

ALHAMDAN, A.; SASTRY, S. K. Natural convection heat transfer between nonNewtonian fluids and an irregular shaped particle, J. Food Process Eng, v.13, p. 113$124,1990$.

ASSIS, T. F. ; ROJAS, E. E. G. ; GUIMARÃES, G. C. ; COELHO, M. C. ; RAMOS, A. V. ; COSTA, B. S. ; COIMBRA, J. S. R. Kinematic Viscosity and Density of Binary and Ternary Mixtures Containing Hydrocolloids, Sodium Chloride, and Water. Int. J. Thermophys., v. 31, p. 513-524, 2010.

BOBBIO, P.A., BOBBIO, F.O. Introdução à química de alimentos. 2.ed. São Paulo: Varela, 1995.

BOWERS, J. Food Theory and Aplications, 2nd Edition, New York: Macmillan Publishing Company, p. 411, 1992.

COSTA, BERNARDO S. ; ROJAS, E. E. G. ; COIMBRA, J. S. R. ; TEIXEIRA, J. A. ; ROMERO, J. T. Density, Refractive Index, Apparent Specific Volume, and Electrical 
Conductivity of Aqueous Solutions of Poly(ethylene glycol) 1500 at Different Temperatures. Chem. Eng. J. Data, v. 59, p. 339-345, 2014.

GUIMARÃES, G. C. ; COELHO JUNIOR, M. C. ;ROJAS, E. E. G. Density and Kinematic Viscosity of Pectin Aqueous Solution. Chem. Eng. J. Data, v. 54, p. 662-667, 2009.

KÄISTNER, U., HOFFMANN, H., DÖNGES R. AND HILBIG, J. Structure and solution properties of sodium carboxymethyl cellulose, Colloids Surf. A., v. 123-124, p.307-328, 1997.

KREVELEN, D. W. V. Properties of polymers. São Paulo, Elsevier, 1990.

MCCABE, W. L.; SMITH, J.C.; HARRIOT, P. Unit operations of chemical engineering, 5 ed., McGraw-Hill, NY, USA, 1993.

PERRECHIL, F.A.; CUNHA, R.L. Development of multiple emulsions based on the repulsive interaction between sodium caseinate and LBG. Food Hydrocolloids, v.26, p. 126-134, 2012.

PHILIPS, G. O.; WILLIANS, P. A.; Handbook of hydrocolloids, 2ed. Woodhead Publishing Limited, 2009.

PILIZOTA, V.; SUBARIC, D.; LOVRIC, T. Rheological properties of CMC dispersions at low temperatures. Food technol. Biotech., v. 34 . p. 87-90, 1996.

RAMOS, A. V. ; ROJAS, E. E. G. ; GIRALDO-ZUNIGA, A. D. Density and Viscosity of Ternary Mixtures of k-Carrageenan, Sodium Chloride, and Water. Int. J. Thermophys., v. 34, p. 240-249, 2013.

THAKUR, B. R. SINGH, R. K.; HANDA, A.V. Chemistry and uses of pectin - A Review. Crit. Rev. Food Sci. Nutr., v.37, n.1, 1997. 